\title{
Constraints on the parameters of radiatively decaying dark matter from the dark matter halos of the Milky Way and Ursa Minor
}

\author{
A. Boyarsky ${ }^{1,2,3}$ J. Nevalainen ${ }^{4}$, and O. Ruchayskiy ${ }^{5,2}$
}

1 CERN, PH-TH, 1211 Geneve 23, Switzerland

e-mail: alexey.boyarsky@epfl.ch

2 École Polytechnique Fédérale de Lausanne, Institute of Theoretical Physics, FSB/ITP/LPPC, BSP 720, 1015 Lausanne, Switzerland

3 On leave of absence from Bogolyubov Institute of Theoretical Physics, Kyiv, Ukraine

4 Helsinki University Observatory, Finland e-mail: jnevalai@astro.helsinki.fi

5 Institut des Hautes Études Scientifiques, 91440 Bures-sur-Yvette, France

e-mail: ruchay@ihes.fr

Received 20 November 2006 / Accepted 19 April 2007

\section{ABSTRACT}

\begin{abstract}
Aims. We improve the earlier restrictions on parameters of the warm dark matter (DM) in the form of a sterile neutrinos. Methods. The results were obtained from not observing the DM decay line in the X-ray spectrum of the Milky Way (using the recent XMM-Newton PN blank sky data). We also present a similar constraint coming from the recent XMM-Newton observation of Ursa Minor - dark, X-ray quiet dwarf spheroidal galaxy.

Results. The new Milky way data improve on (by as much as the order of magnitude at masses $\sim 3.5 \mathrm{keV}$ ) existing constraints. Although the observation of Ursa Minor has relatively poor statistics, the constraints are comparable to those recently obtained using observations of the Large Magellanic Cloud or M 31. This confirms a recent proposal that dwarf satellites of the MW are very interesting candidates for the DM search and dedicated studies should be made to this purpose.
\end{abstract}

Key words. Galaxy: halo - galaxies: individual: Ursa Minor - cosmology: dark matter - X-rays: galaxies

\section{Introduction}

This past year has seen a lot of activity, devoted to searching for the decay signals of the DM particle in X-ray spectra of various astrophysical objects (Boyarsky et al. 2006a,b,c; Riemer-Sørensen et al. 2006a; Watson et al. 2006; Riemer-Sørensen et al. 2006b, e.g). It was noticed long ago by Dodelson \& Widrow (1994) that a right-handed neutrino with masses in the $\mathrm{keV}$ range presents a viable warm dark matter (WDM) candidate. Such a particle possesses a specific radiative decay channel, so one can search for its decay line in the X-ray spectra of astrophysical objects (Dolgov \& Hansen 2002; Abazajian et al. 2001b).

Recently, the interest in the sterile neutrino as a DM candidate has been greatly revitalized. First, the discovery of neutrino oscillations (see e.g. Strumia \& Vissani 2006, for a review) strongly suggest the existence of right-handed neutrinos. Probably the easiest way to explain the data on oscillations is by adding several right-handed, or sterile, neutrinos, to the Standard Model. It has been demonstrated recently in Asaka et al. (2005) and Asaka \& Shaposhnikov (2005) that a simple extension of the Standard Model by three singlet fermions with masses smaller than the electroweak scale (dubbed the $v$ MSM in Asaka et al. 2005) allows accommodation of the data on neutrino masses and mixings, allows baryon asymmetry of the Universe to be explained, and provides a candidate for the dark matter particle in the form of the lightest of the sterile neutrinos ${ }^{1}$.

1 The $v$ MSM does not explain the unconfirmed results of the LSND experiment (Aguilar et al. 2001). There are other models that try
Secondly, warm DM with the mass of particle in $\mathrm{keV}$ range can ease the problem of the dark halo structures in comparison with the cold dark matter scenario (Bode et al. 2001; Goerdt et al. 2006). By determining the matter power spectrum from the Lyman- $\alpha$ forest data from SDSS Seljak et al. (2006) and Viel et al. (2006) argue that the mass of the DM particles should be in the range $\gtrsim 10 \mathrm{keV}$ ( $\gtrsim 14 \mathrm{keV}$ in the case of Seljak et al. 2006). As this method gives direct bounds for the free-streaming length of the neutrinos, the bounds on the mass of the DM particle depend on the momentum distribution function of the sterile neutrinos and, therefore, on their production mechanism. The results quoted above are claimed for the simplest Dodelson-Widrow model (1994).

At the same time, studies of the Fornax dwarf spheroidal galaxy (Goerdt et al. 2006; Strigari et al. 2006) disagree with the predictions of CDM models and suggest lower mass than in Seljak et al. (2006) and Viel et al. (2006) for the DM particle $M_{\mathrm{DM}} \sim 2 \mathrm{keV}$. This result agrees with the earlier studies of Hansen et al. (2002) and Viel et al. (2005), which used a different dataset. For other interesting applications of the sterile neutrinos with the mass $\sim$ few keV see e.g. Kusenko (2006b), Biermann \& Kusenko (2006), Stasielak et al. (2006), Kusenko (2006a), and Hidaka \& Fuller (2006).

to account for it by introducing a sterile neutrino with the mass around $1 \mathrm{eV}$. There are also models that explain not all, but only some of these phenomena (e.g. LSND and DM, but not the baryon asymmetry as e.g. in de Gouvea 2005) We do not give any review here. We would like to stress that, although our work is motivated by the recent results on the $v$ MSM, our method and results do not rely on any particular model. 
It has been argued in Boyarsky et al. (2006c) and Riemer-Sørensen et al. (2006a) that the preferred targets for observations are objects from the local halo, including our own Milky Way and its satellites. In particular, Boyarsky et al. (2006c) showed that the best observational targets are the dwarf spheroidals (Ursa Minor, Draco, etc.). Indeed, these objects are $\mathrm{X}$-ray quiet, while at the same time one expects the DM decay signal from them, comparable to what comes from galaxy clusters. Because at the time of writing of Boyarsky et al. (2006c) no public data were available for these dwarf spheroidals, the observations of the core of Large Magellanic Cloud (LMC) were used to produce the strongest restrictions on parameters of the sterile neutrino. It was stressed in Boyarsky et al. (2006c) that other dwarf satellite galaxies should be studied as well, in order to minimize uncertainties related to the DM modeling in each single object. In this paper we continue studies of the dwarf satellites of the MW by analyzing the data from XMM-Newton observation of Ursa Minor and confirm the restrictions of Boyarsky et al. (2006c).

It was also shown in Boyarsky et al. (2006c) that the improvement of the results from MW DM halo can be achieved by using longer exposure data (notably, longer exposure of the closed filter observations). In this paper, we improve our restrictions, coming from the MW DM halo by using the blank sky dataset with better statistics from Nevalainen et al. (2005).

\section{DM with radiative decay channel}

Although throughout this paper we are talking mostly about the sterile neutrino DM, the results can be applied to any DM particle that possesses the monoenergetic radiative decay channel, emits photon of energy $E_{\gamma}$ and has a decay width $\Gamma$. In the case of the sterile neutrino (with mass below that of an electron), the radiative decay channel is into a photon and active neutrino $\mathrm{Pal}$ $\&$ Wolfenstein (1982). As the mass of an active neutrino is much lower than $\mathrm{keV}, E_{\gamma}=\frac{M_{\mathrm{s}}}{2}$ in this case. The width $\Gamma$ of radiative decay can be expressed (Pal \& Wolfenstein 1982; Barger et al. $1995)$ in terms of mass $M_{\mathrm{s}}$ and mixing angle $\theta$ via

$\Gamma=\frac{9 \alpha G_{F}^{2}}{1024 \pi^{4}} \sin ^{2} 2 \theta M_{\mathrm{s}}^{5} \simeq 1.38 \times 10^{-22} \sin ^{2}(2 \theta)\left[\frac{M_{\mathrm{s}}}{\mathrm{keV}}\right]^{5} \mathrm{~s}^{-1}$.

(The notation $\sin ^{2}(2 \theta)$ is used traditionally, although in all realistic cases $\theta \ll 1$.) The flux of the DM decay from a given direction is given by

$F_{\mathrm{DM}}=\Gamma \frac{E_{\gamma}}{M_{\mathrm{s}}} \int_{\text {fov cone }} \frac{\rho_{\mathrm{DM}}(\boldsymbol{r})}{4 \pi\left|\boldsymbol{D}_{L}+\boldsymbol{r}\right|^{2}} \mathrm{~d}^{3} \boldsymbol{r}$.

Here $\boldsymbol{D}_{L}$ is the luminous distance between the observer and the center of the observed object, $\rho_{\mathrm{DM}}(r)$ is the DM density, and the integration is over the DM distribution inside the (truncated) cone - solid angle, spanned by the field of view (FoV) of the $\mathrm{X}$-ray satellite. If the observed object is $\mathrm{far}^{2}$, then Eq. (2) can be simplified:

$F_{\mathrm{DM}}=\frac{M_{\mathrm{DM}}^{\mathrm{fov}} \Gamma}{4 \pi D_{L}^{2}} \frac{E_{\gamma}}{M_{\mathrm{s}}}$,

where $M_{\mathrm{DM}}^{\mathrm{fov}}$ is the mass of DM within a telescope's field of view (FoV). Equation (3) can be rewritten again as

$F_{\mathrm{DM}}=6.38 \times 10^{6}\left(\frac{M_{\mathrm{DM}}^{\text {fov }}}{10^{10} M_{\odot}}\right)\left(\frac{\mathrm{kpc}}{D_{L}}\right)^{2} \times \sin ^{2}(2 \theta)\left[\frac{M_{\mathrm{s}}}{\mathrm{keV}}\right]^{5} \frac{\mathrm{keV}}{\mathrm{cm}^{2} \cdot \mathrm{s}} .(4)$

${ }^{2}$ Namely, if luminosity distance $D_{L}$ is much greater than the characteristic scale of the DM distribution $\rho_{\mathrm{DM}}(r)$.
In the absence of a clearly detectable line, one can put an upper limit on the flux of DM from the astrophysical data, which will lead via Eq. (4) to the restrictions of parameters of the sterile neutrino $M_{\mathrm{s}}$ and $\theta$.

\section{Restrictions from the blank sky observation}

\subsection{Modeling the DM halo of the MW}

As shown in the previous section, one needs to know the distribution of the DM to obtain the restrictions on parameters of the sterile neutrino. In the case of nearby objects (including our own Galaxy and dwarf satellites from the local halo), the DM distribution can be deduced e.g. by using the rotation curves of the stars in the galaxy. Here we follow the analysis of Boyarsky et al. (2006c). Various DM profiles, used to fit observed velocity distributions, differ the most in the center of a distribution. In the case of the MW we choose, as in Boyarsky et al. (2006c), to use the observations away from the center, to minimize this uncertainty. In particular, in references Klypin et al. (2002) and Battaglia et al. (2005) it was shown that the DM halo of the MW can be described by the Navarro-Frenk-White (NFW) profile (Navarro et al. 1997)

$\rho_{\mathrm{NFW}}(r)=\frac{\rho_{\mathrm{s}} r_{\mathrm{s}}^{3}}{r\left(r+r_{\mathrm{s}}\right)^{2}}$,

with parameters, given in Table $1^{3}$. The relation between virial parameters and $\rho_{\mathrm{s}}, r_{\mathrm{s}}$ are given in the Appendix A. Quoted halo parameters provide DM decay flux (from the directions with $\phi>90^{\circ}$ ) consistent within $\sim 5 \%$ with the one, given by Eqs. (6), (7). Only "maximal disk" models in Klypin et al. (2002) would provide 30-50\% weaker restrictions; however, these models are highly implausible, see Klypin et al. (2002). Similarly, taking the lower limit for the virial mass of Battaglia et al. (2005), one would obtain $25 \%$ weaker restrictions than the ones, presented in this paper 4 .

To compare the results from different (e.g. cuspy and cored) profiles, we can also describe the DM distribution in the MW via an isothermal profile:

$\rho_{\text {iso }}(r)=\frac{v_{\mathrm{h}}^{2}}{4 \pi G_{\mathrm{N}}} \frac{1}{r^{2}+r_{\mathrm{c}}^{2}}$.

The DM flux from a given direction $\phi$ into the solid angle $\Omega_{\text {fov }} \ll$ 1 , measured by an observer on Earth (distance $r_{\odot} \simeq 8 \mathrm{kpc}$ from the galactic center), is given by

$F_{\mathrm{DM}}^{\text {iso }}(\phi)=\frac{L_{0}}{R} \times \begin{cases}\frac{\pi}{2}+\arctan \left(\frac{r_{\odot} \cos \phi}{R}\right), & \cos \phi \geq 0 \\ \arctan \left(\frac{R}{r_{\odot}|\cos \phi|}\right), & \cos \phi<0 .\end{cases}$

Here $L_{0} \equiv \frac{\Gamma \Omega_{\mathrm{fov}} v_{\mathrm{h}}^{2}}{32 \pi^{2} G_{\mathrm{N}}}$ and $R=\sqrt{r_{\mathrm{c}}^{2}+r_{\odot}^{2} \sin ^{2} \phi}$. Angle $\phi$ is related to the galactic coordinates $(b, l)$ via

$\cos \phi=\cos b \cos l$.

${ }^{3}$ According to Klypin et al. (2002), the choice of e.g. the Moore profile Ghigna et al. (2000) or a generalization thereof, as compared to the NFW profile, would change the results by $\lesssim 1 \%$ for $r<3 \mathrm{kpc}$. As we are using observations away from the center, this difference is completely negligible, so we choose to use the NFW profile.

4 When quoting results of Klypin et al. (2002), we do not take the effects of baryon compression on DM into account. While these effects make DM distribution in the core of the MW denser, they are hard to compute precisely. Thus the values we adopt give us a conservative lower bound on the estimated DM signal. 
Table 1. Best-fit parameters of NFW model of the MW DM halo.

\begin{tabular}{cccccc}
\hline \hline References & $M_{\text {vir }}\left[M_{\odot}\right]$ & $r_{\text {vir }}[\mathrm{kpc}]$ & Concentration & $r_{\mathrm{s}}[\mathrm{kpc}]$ & $\rho_{\mathrm{s}}\left[M_{\odot} / \mathrm{kpc}^{3}\right]$ \\
\hline Klypin et al. (2002), favored models $\left(A_{1}\right.$ or $\left.B_{1}\right)$ & $1.0 \times 10^{12}$ & 258 & 12 & 21.5 & $4.9 \times 10^{6}$ \\
Battaglia et al. (2005) & $0.8_{-0.2}^{+1.2} \times 10^{12}$ & 255 & 18 & 14.2 & $11.2 \times 10^{6}$ \\
\hline
\end{tabular}

Thus, the galactic center corresponds to $\phi=0^{\circ}$, and the anticenter $\phi=180^{\circ}$ and the direction perpendicular to the galactic plane to $\phi=90^{\circ}$.

In Boyarsky et al. (2006c), the following parameters of isothermal profile were chosen: $v_{\mathrm{h}}=170 \mathrm{~km} \mathrm{~s}^{-1}$ and $r_{\mathrm{c}}=4 \mathrm{kpc}$. One can easily check (using Table 1 and Eqs. (A.5), (A.6) in Appendix A) that, in the directions $\phi \gtrsim 90^{\circ}$, the difference in predicted DM fluxes between the NFW model with parameters, given in Table 1 and isothermal model with parameters just quoted are completely negligible (less than $5 \%)^{5}$.

\subsection{XMM-Newton PN blank sky data}

To examine the Milky Way halo, we used the double-filtered, single+double event XMM-Newton PN blank sky data from Nevalainen et al. (2005), which is a collection of 18 blank sky observations (see Table 2 in Nevalainen et al. 2005, for their observation IDs, positions, and exposures) ${ }^{6}$. The exposure time of the co-added observations is $547 \mathrm{ks}$. We used a combination of closed-filter observations from Nevalainen et al. (2005) (total exposure time $145 \mathrm{ks}$ ) to model the background of XMM-Newton PN instrument separately. The data has been filtered using SAS expression "flag $==0$ ", which rejects the data from bad pixels and CCD gap regions. After removing the brightest point sources, the total accumulation area is $603 \operatorname{arcmin}^{2}$.

Based on the $>10 \mathrm{keV}$ band count rates of the blank sky and the closed filter data, we normalized the closed-filter spectrum by a factor of 1.07 before subtracting it from the blank sky spectrum. The remaining sky-background spectrum consists mainly of the Galactic emission and the cosmic X-ray background $(\mathrm{CXB})$ due to unresolved extragalactic point sources. We modeled the Galactic emission by a non-absorbed MEKAL model with Solar abundances. For the CXB emission, we used a power-law model modified at the lowest energies by Galactic absorption with the value of $N_{\mathrm{H}}$ fixed to its exposure-weighted average over all blank sky observations $\left(N_{\mathrm{H}}=1.3 \times 10^{20} \mathrm{~cm}^{-2}\right)$.

The variable Galactic emission and geocoronal Solar windcharge exchange emission (see e.g. Wargelin et al. 2004) complicate the modeling at the lowest energies. The remaining calibration uncertainties further complicate the analysis in the

5 Abazajian \& Koushiappas (2006) claim that the MW results of Boyarsky et al. (2006c); Riemer-Sørensen et al. (2006a) are uncertain by about a factor of 3 . This conclusion was based on the range of virial masses of the MW DM halo $M_{\mathrm{vir}}=(0.7-2.0) \times 10^{12} M_{\odot}$ in Klypin et al. (2002). However, as just demonstrated, the authors of Boyarsky et al. (2006c) have chosen parameters of DM halo conservatively. The flux they used, corresponded to the favored models $A_{1}$ or $B_{1}$ in Klypin et al. (2002), with $M_{\text {vir }} \sim 1.0 \times 10^{12} M_{\odot}$. These models provide the lowest bound on the derived flux of DM decay (if one does not take into account the highly implausible "maximum disk" $\left(A_{2}\right.$ or $\left.B_{2}\right)$ models of Klypin et al. 2002). Even in the latter case, the DM flux will be 30-50\% lower than the one used in work Boyarsky et al. (2006c). Therefore, parameters of the MW DM halo from Boyarsky et al. (2006c) provide the conservative estimate so we use them in our work as well.

${ }^{6}$ We processed the blank sky data with newer SAS distribution, xmmsas_20050815_1803-6.5.0, and obtained slightly different exposure times than those in the public data.

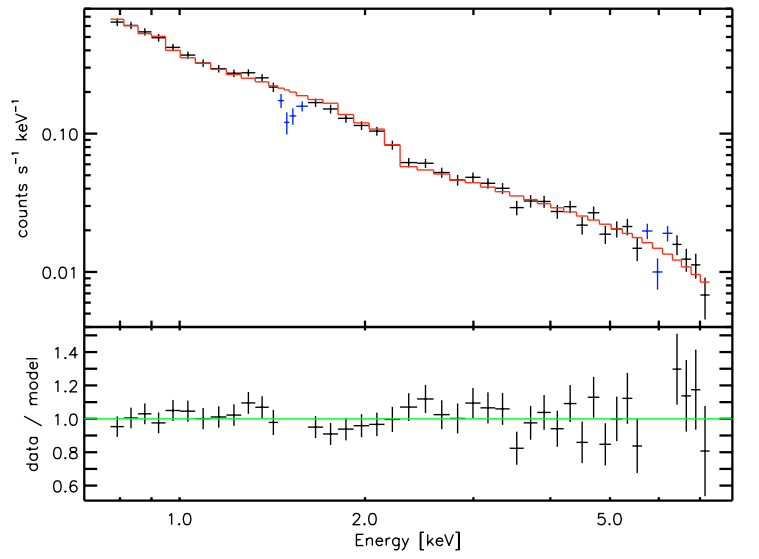

Fig. 1. Upper panel: the blank sky data of Nevalainen et al. (2005) after subtraction of the closed filter data. The black crosses show the data points, used for modeling the Galactic emission and CXB. The blue crosses show the data points excluded from the fit. The best-fit model is shown with the red line. The plotted spectrum is binned by a minimum of 20000 counts per channel, different from that used in the actual fit (see text). The error bars include the 5\% systematic uncertainty used in the analysis. Lower panel: the ratio of the data-to-model values in each channel used in the fit.

lowest energies (see e.g. Nevalainen et al. 2006). Thus, we omitted the channels below $0.8 \mathrm{keV}$. At energies above $7 \mathrm{keV}$, the particle background dominates and the total flux is very sensitive to the background normalization. We thus excluded channels above $7 \mathrm{keV}$.

The data are not well-described in the 1.45-1.55 and $5.8-6.3 \mathrm{keV}$ bands with the above model. These deviations probably originate from the variability of the instrumental $\mathrm{Al}$ and Fe line emission. In order to minimize the effect of the instrumental problems, we excluded these bands when finding the best-fit sky background model (see below). Also, to account for possibly remaining calibration inaccuracies, we added a systematic uncertainty of $5 \%$ of the model value in each bin in quadrature to the statistical uncertainties.

We binned the spectrum using a bin size of $1 / 3$ of the energy resolution and fitted the data using models and channels as described above. The best-fit (reduced $\chi^{2}=1.03$ for 153 degrees of freedom) model agrees with the data within the uncertainties (see Fig. 1), yielding a photon index of $1.50 \pm 0.02$ at $1 \sigma$ confidence level (see Fig. 1), consistent with similar analyses based on Chandra (Hickox \& Markevitch 2006) and XMM-Newton MOS instrument (De Luca \& Molendi 2004). The best-fit temperature of the MEKAL component used to model the Galactic emission is $0.19 \pm 0.01 \mathrm{keV}$, consistent with e.g. Hickox \& Markevitch (2006).

We then evaluated the level of possible DM flux above the background model allowed by the statistical and systematic uncertainties in each channel. For this, we modified the above bestfit model by adding a narrow (width $=1 \mathrm{eV}$ ) Gaussian line to it. We then re-fitted the data, fixing the Gaussian centroid for each fit to the central energy of a different channel. In these fits we fixed the above continuum model parameters to the best-fit 


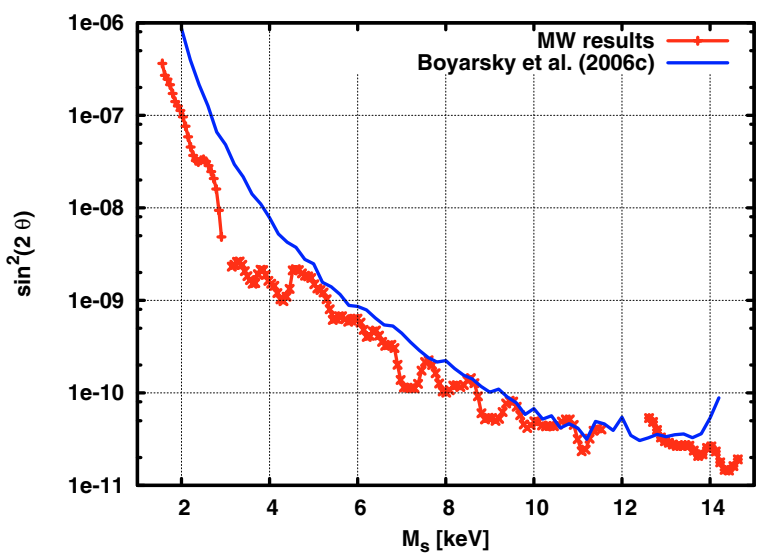

Fig. 2. Exclusion plot based on the blank sky observations. Bins $2.9-3.1 \mathrm{keV}$ and $11.6-12.6 \mathrm{keV}$ are excluded.

values and thus the Gaussian normalization parameter is the only free parameter. We used the fits to find the upper $3 \sigma$ uncertainty of the Gaussian normalization, i.e. the allowed DM flux. Note that here we included the channels $1.45-1.55$ and $5.8-6.3 \mathrm{keV}$ (excluded above when defining the sky background model). The background is oversubtracted in the channels at $1.45-1.55 \mathrm{keV}$ and $\sim 6.0 \mathrm{keV}$ (see Fig. 1), which would formally require negative normalization for the Gaussian. However, we forced the normalization to be positive and thus obtained conservative upper limits in these energies.

Finally, we converted the upper bound obtained for the flux per energy bin to the restrictions on $M_{\mathrm{s}}$ and $\sin ^{2} 2 \theta$, using Eqs. (1), (7) (we use exposure weighted average of DM fluxes (Eq. (7)) from all the observations, constituting the blank sky dataset). This corresponds to the average "column density" $1.22 \times 10^{28} \mathrm{keV} / \mathrm{cm}^{2}$. The results are shown in Fig. 2

At energies above $E=5 \mathrm{keV}$, the instrumental background of PN dominates over the sky background (cf. Nevalainen et al. 2005). Therefore, the accuracy of the co-added closed filter spectrum in predicting the particle background in the blank sky observations becomes essential. We estimate this accuracy using the variability of the individual closed-filter spectra in the $0.8-7.0 \mathrm{keV}$ band (Nevalainen et al. 2005) and propagate it by varying the normalization of closed filter data by $\pm 5 \%$ and repeating the above analysis. This leads to a factor of 3 change in the results at $M_{\mathrm{s}} \sim 14 \mathrm{keV}$ (see Fig. 3). Therefore, for $E \gtrsim 5 \mathrm{keV}$ we choose the more conservative normalization (see Fig. 7 below).

\section{Restrictions from observations of Ursa Minor}

It was argued in Boyarsky et al. (2006c) that dwarf satellite galaxies should provide the best restrictions, based on their high concentration of DM and low X-ray signal. At the moment of writing of Boyarsky et al. (2006c), no public data on preferred dwarf satellites were available, therefore the observation of core of LMC were used as a demonstration. Recently, the Ursa Minor dwarf (UMi) was observed with XMM-Newton (obs IDs.: 0301690201, 0301690301, 0301690401, 0301690501, observed in August-September 2005) ${ }^{7}$. Unfortunately, most of these observations are strongly contaminated by background flares and the observations have very small exposure times. Below we present the analysis of only one observation

7 We are very grateful to Prof. T. Maccarone for sharing this data with us before it became publicly available through the XMM data archive.

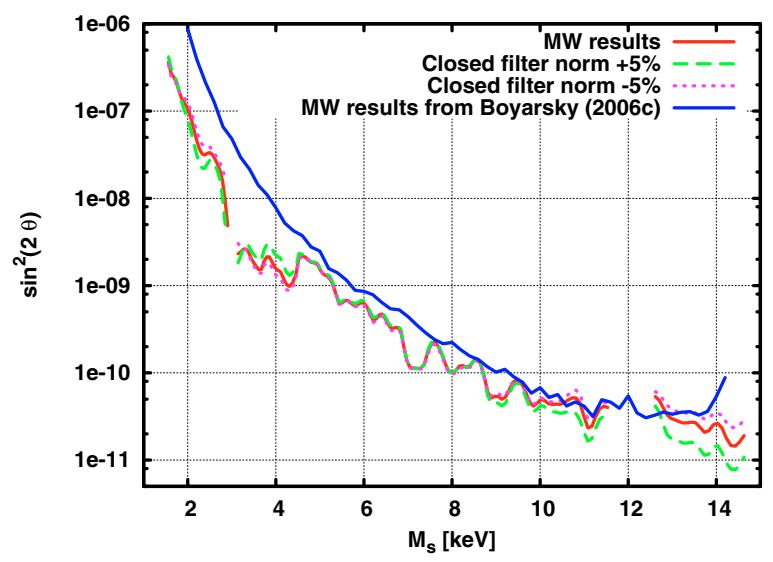

Fig. 3. Dependence of the results on closed filter normalization. Red (solid) and green (long-dashed) lines are the same as in Fig. 2.

(obsID: 0301690401), which "suffered" the least from background contamination.

\subsection{DM modeling for UMi}

The DM distribution in UMi has a cored profile (see e.g. Kleyna et al. 2003; Wilkinson et al. 2006; Gilmore et al. 2006, 2007) ${ }^{8}$. We adopt the following parameters of isothermal profile (6) for UMi: $v_{\mathrm{h}}=23 \mathrm{~km} \mathrm{~s}^{-1}, r_{\mathrm{c}}=0.1 \mathrm{kpc}$ (see e.g. Wilkinson et al. 2006) ${ }^{9}$. We adopt the distance to UMi $D_{L}=66 \mathrm{kpc}$ (Mateo 1998). The DM mass within the circular FoV with the radius $r_{\text {fov }}$, centered at the center of the galaxy is given by

$M_{\mathrm{DM}}^{\mathrm{fov}}=\frac{\pi v_{\mathrm{h}}^{2}}{2 G_{\mathrm{N}}}\left(\sqrt{r_{\mathrm{fov}}^{2}+r_{\mathrm{c}}^{2}}-r_{\mathrm{c}}\right)$.

In our case, the radius of FoV is $13.9^{\prime}$, which corresponds to $r_{\text {fov }}=0.27 \mathrm{kpc}$ (i.e. about $3 r_{\mathrm{c}}$ ). Therefore

$M_{\mathrm{DM}}^{\mathrm{fov}}=3.3 \times 10^{7} M_{\odot}$ for $r_{\mathrm{fov}}=0.27 \mathrm{kpc}$.

Using Eqs. (10) and (3), one can compute the expected DM flux from UMi:

$F_{\mathrm{DM}}=4.79 \frac{\mathrm{keV}}{\mathrm{cm}^{2} \cdot \mathrm{s}}\left[\frac{M_{\mathrm{s}}}{\mathrm{keV}}\right]^{5} \sin ^{2}(2 \theta)$.

\subsection{PN data analysis}

We processed the Ursa Minor observation 0301690401 using epchain version 8.56 and filtered the event file with SAS

\footnotetext{
${ }^{8}$ As discussed in Sect. 3.1, the estimates for DM flux do not vary significantly if one uses NFW instead of the isothermal DM density profile. In the case of UMi, the cored (isothermal) profile will clearly produce a more conservative estimate than will the cuspy NFW profile. Indeed, taking NFW parameters for UMi from the recent paper $\mathrm{Wu}$ (2007) gives a $\sim 20 \%$ higher estimate for the DM mass within the FoV.

${ }^{9}$ For the detailed studies of mass distribution in dwarf spheroidals, see Gilmore et al. (2007). We are grateful to Prof. G. Gilmore for sharing the numbers with us before their paper became available. The statistical uncertainty in determining these numbers is below $10 \%$. The systematic uncertainties are much harder to estimate. One of the major sources of the systematic errors comes from violation of the main assumptions of the method: deviation from equilibrium and from the spherical distribution of matter in a galaxy. In other known examples it provides a factor of 2 uncertainty, which should be a conservative estimate in the case of UMi, as it is rather spherical. Another typical uncertainty - determination of the mass of the stars - is not important for UMi, as it has a very high mass-to-light ratio.
} 


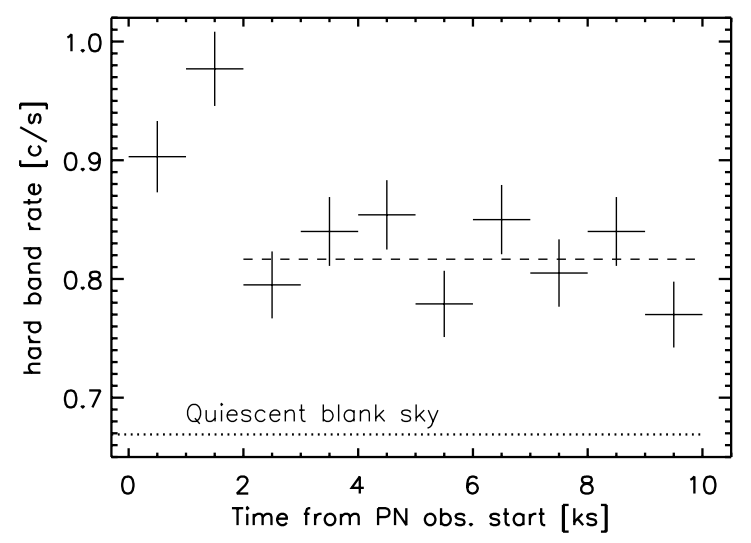

Fig. 4. Hard band light curve for the UMi observation 0301690401. The crosses show the PN $>10 \mathrm{keV}$ band rate of the full FOV in $1 \mathrm{ks}$ bins. The dashed line shows the average, when excluding first 2 time bins. The dotted line shows the corresponding quiescent value in the coadded blank sky data (Nevalainen et al. 2005).

expressions "PATTERN $<=4$ " and "FLAG==0". We applied the blank sky-based XMM-Newton background method of Nevalainen et al. (2005) for Ursa Minor. The $>10 \mathrm{keV}$ band light curve from the full FOV (Fig. 4) shows that the count rate in observation 0301690401 (excluding first $2 \mathrm{ks}$ ) exceeds that of the blank sky quiescent average by $25 \%$. This level is higher, but close to what is used in the blank sky accumulation $( \pm 20 \%$ filtering around the quiescent level). Thus we accepted the data from all instants after the initial $2 \mathrm{ks}$, and we approximated the background uncertainties with those in Nevalainen et al. (2005).

The hydrogen column density in the direction of Ursa Minor is small $\left(\sim N_{\mathrm{H}}=2 \times 10^{20} \mathrm{~cm}^{-2}\right)$ and consistent with the variation in the blank sky sample. Thus we can also apply the blank sky background method to channels below $2 \mathrm{keV}$.

As noted in the above XMM-Newton blank sky study, the $>10 \mathrm{keV}$ band-based scaling of the background only works up to a factor of 1.1, beyond which the background prediction becomes worse. Furthermore, the correlation of background rates in the $>10 \mathrm{keV}$ band is very poor with the rates below $2 \mathrm{keV}$ band. Thus, in order to achieve the best possible background prediction accuracy, we scaled the blank sky background spectrum by a factor of 1.1 at channels above $2 \mathrm{keV}$, and we applied no background scalingat lower energies.

We removed this scaled background spectrum from the Ursa Minor spectrum (see Fig. 5). As shown in Nevalainen et al. (2005), the background accuracy is worse at lower energies. We used those estimates to propagate the background uncertainties at $1 \sigma$ confidence level to our results by examining how the results change when varying the $0.8-2.0 \mathrm{keV}$ and $2.0-7.0 \mathrm{keV}$ band background by $15 \%$ and $10 \%$, respectively.

\subsection{Ursa Minor data and restrictions on the sterile neutrino parameters}

The X-ray spectrum of UMi is similar to that of LMC: above $2 \mathrm{keV}$ the flux is zero within statistical limits (see Fig. 5). (Of course, the data set has rather low statistics: after the cleaning of flares the UMi observation only contains $7 \mathrm{ks}$.) Therefore, for such data, we utilized the "total flux" method. Namely, we restricted the DM flux in the given energy bin to be bounded from above by the measured total flux in this energy bin plus its $3 \sigma$ uncertainty (Fig. 5). As each energy bin has a width of twice the FWHM at a given energy, the flux from a DM line would not

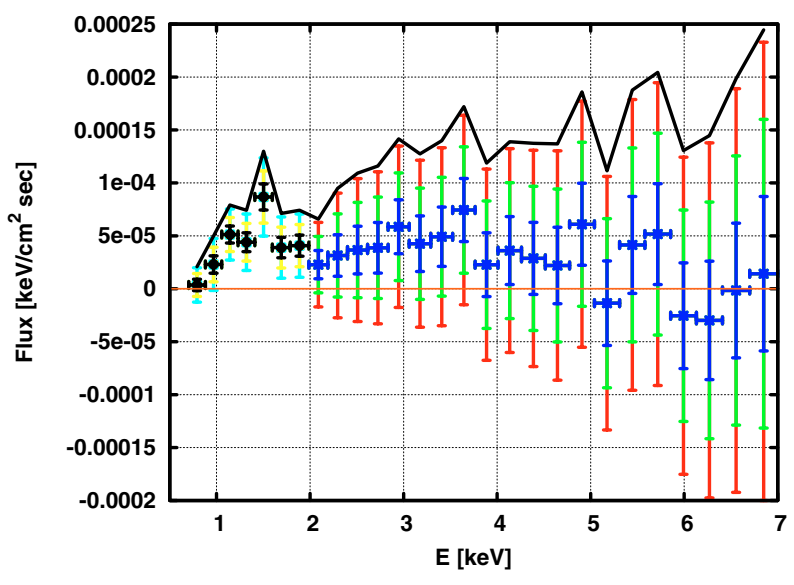

Fig. 5. Flux from UMi (obs. ID 0301690401). Energy bins have the width of twice the spectral resolution. Shown are the 1,2 , and $3 \sigma$ errors. One can see that, above $2 \mathrm{keV}$, flux in most energy bins is zero within $1 \sigma$ limits (blue crosses) and for the rest it is zero within $2 \sigma$ limits (green crosses). Similarly, below $2 \mathrm{keV}$ black, cyan and yellow crosses represent 1,2 , and $3 \sigma$ error correspondingly. The solid black line represents the $3 \sigma$ upper bound on total flux in a given energy bin, which we use to put the limit on DM parameters.

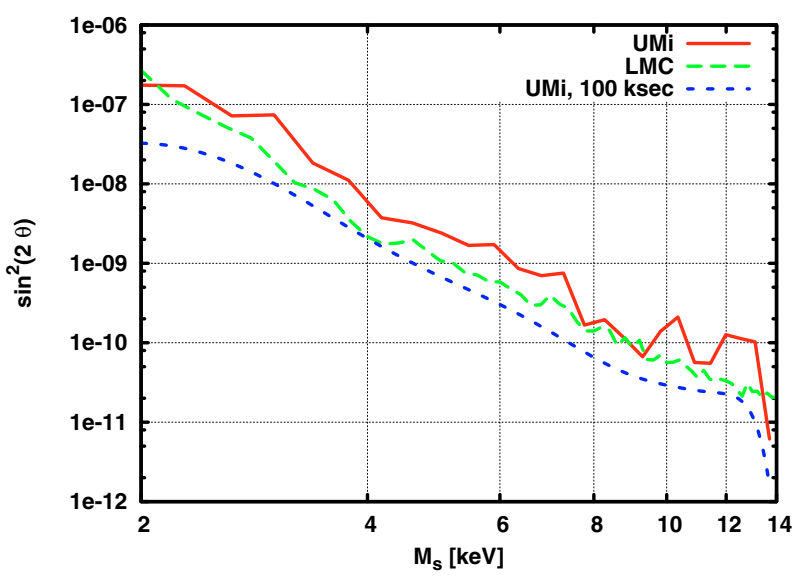

Fig. 6. Exclusion from UMi (red solid line), as compared to LMC (green long-dashed line). The blue short-dashed smooth curve shows hypothetical restrictions from UMi observations with $100 \mathrm{ks}$ exposure.

"spill" into nearby bins. Using Eq. (11), we find the restrictions on the sterile neutrino parameters, represented in Fig. 6.

These restrictions should be compared with those, obtained from another satellite galaxy - Large Magellanic Cloud (Boyarsky et al. 2006c). As one clearly sees from Fig. 6, in spite of the low exposure time, it is fully consistent with the earlier bounds from LMC, thus confirming the results of Boyarsky et al. (2006c). Improvement of the exposure for UMi observations should, presumably lead to the improvement of results (at least for energies above $E \gtrsim 2 \mathrm{keV}$ ). For example, for a $100 \mathrm{ks}$ observation, we expect the results to improve by roughly a factor $\sqrt{100 \mathrm{ks} / 7 \mathrm{ks}} \approx 3.77$.

\section{Results}

\subsection{Restrictions from the blank sky data}

By analyzing the blank sky data set with better statistics, we improved on the previous results of Boyarsky et al. (2006c), Riemer-Sørensen et al. (2006a), Watson et al. (2006) (by as 


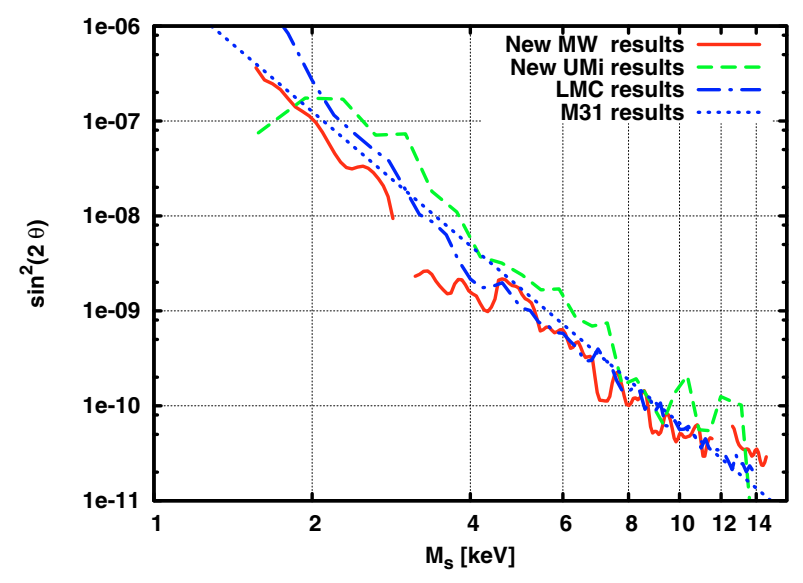

Fig. 7. Results and comparison with previous bounds (a region of parameter space above a curve is excluded).

much as the factor of 10 for $M_{\mathrm{s}} \approx 3.5 \mathrm{keV}$ and by the negligible amount for $M_{\mathrm{s}} \gtrsim 11 \mathrm{keV}$ ). The result is shown in Fig. 7 in red solid line. The best previous bounds are also shown: bound from LMC (Boyarsky et al. 2006c) with a blue short-dashed line and bound from M 31 (Watson et al. 2006) with a dotted magenta line. We see that in the region $3.5 \mathrm{keV} \lesssim M_{\mathrm{s}} \lesssim 11 \mathrm{keV}$ the new blank sky data improves on previous results. These results can be converted (using Eq. (1)) into restrictions on the decay rate $\Gamma$ of any DM particle, that possesses radiative decay channel and emits a photon $E_{\gamma}$ (see Fig. 8). Our results provide more than an order of magnitude improvement over similar restrictions derived in Riemer-Sørensen et al. (2006a) (which used the Chandra blank sky background), as one can clearly see by comparing Fig. 8 with Fig. 2 in Riemer-Sørensen et al. (2006a), where the exclusion plot is above $\Gamma=10^{-26} \mathrm{~s}^{-1}$ line for all energies. (In Riemer-Sørensen et al. 2006a, the restriction were made, based on the total flux of Chandra satellite, without subtraction of the instrumental background, which explains a much weaker restrictions.)

The empirical fit to the MW data is given by the following expression:

$\sin ^{2}(2 \theta) \lesssim 2.15 \times 10^{-7}\left(\frac{M_{\mathrm{s}}}{\mathrm{keV}}\right)^{-3.45}$.

\subsection{Restrictions from Ursa Minor dwarf}

Restrictions from XMM observation of UMi are shown in Fig. 7 by the green long-dashed line. These results are slightly weaker than LMC or M 31 results, which is due to the very low statistics of the UMi observation. Improvement of the statistics should lead to improvement of the current bound (as shown in Fig. 6). These results confirm the recent claims (Boyarsky et al. 2006c) that dwarfs of the local halo are promising candidates for the DM-decay line search and, as such, should be studied dedicatedly.

In searching for the DM signatures, it is important to understand that the uncertainties of the DM modeling for any given object can be large, and therefore it is important to study many objects of given type, as well as many different types of objects (where DM distributions are deduced by independent methods). To this end, although UMi data does not provide any improvement over existing bounds, it makes those bounds more robust as the existence of DM in UMi is deduced by independent observations, and the rotation curves of UMi are measured

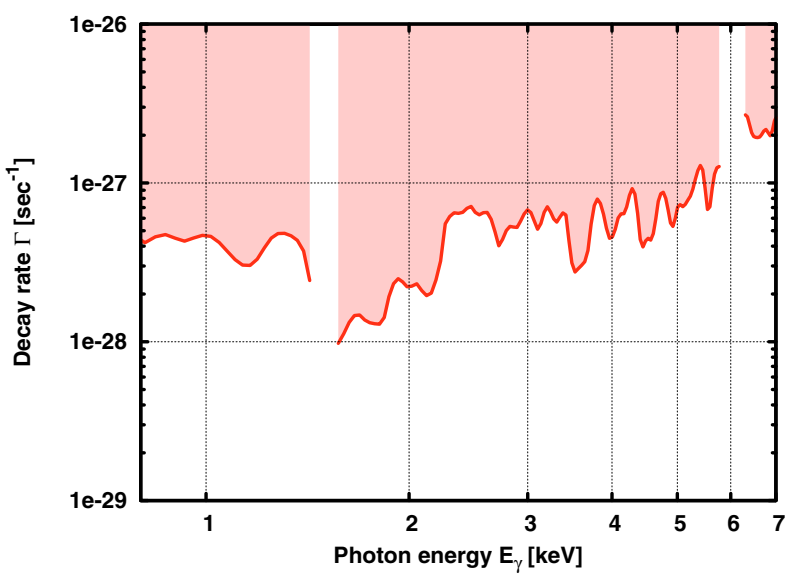

Fig. 8. Restrictions on parameters of any DM particle with the radiative decay width $\Gamma$, emitting photon of energy $E_{\gamma}$. Shaded region is excluded.

quite well, since it has less perturbed dynamics, compared to e.g. LMC.

\section{Discussion}

In this paper we continued to search for the best astrophysical objects, from the point of view of restricting parameters of DM particles with the radiative decay channel. Several comments are in order here.

(1) Although throughout this paper we have spoken about the sterile neutrinos and restricted their parameters (namely, mass, and mixing angle), the constraints can be readily converted into any other DM candidate that possesses a radiative decay channel ${ }^{10}$. Then the restrictions are formulated on the decay rate $\Gamma$ as a function of energy of the emitted photon $E_{\gamma}$. The results then can be presented in the form of an exclusion plot, presented in Fig. 8.

(2) Clearly, if one could relate parameters of the sterile neutrino with their relic abundance $\Omega_{\mathrm{s}}$, this would allow one to put an upper limit on the mass of the sterile neutrino. Unfortunately, such a computation is strongly modeldependent. In Dodelson \& Widrow (1994), Dolgov \& Hansen (2002), Abazajian et al. (2001a), Abazajian (2006), the relic abundance of the sterile neutrinos was computed in a simple model with only one sterile neutrino, assuming the absence of the sterile neutrinos above the temperatures $\sim 1 \mathrm{GeV}$. Yet, even the computation in this simplest model is subject to a number of uncertainties (Shi \& Fuller 1999; Boyarsky et al. 2006a,c; Asaka et al. 2006a,b; Shaposhnikov \& Tkachev 2006). In particular, in Dodelson \& Widrow (1994), Dolgov \& Hansen (2002), Abazajian et al. (2001a), Abazajian (2006), two assumptions were made: (i) the absence of heavy particles, whose decay can dilute the relic abundance, (ii) the absence of lepton asymmetries. In addition, simplifying assumptions about dynamics of hadrons at temperatures $O(150) \mathrm{MeV}$ were used. Recently, Asaka et al. (2007) performed this computation from the first principles, showing that the uncertainty due to QCD effects (between

${ }^{10}$ For earlier works, discussing cosmological and astrophysical effects of decaying DM, see e.g. de Rujula \& Glashow (1980); Berezhiani et al. (1987); Doroshkevich et al. (1989); Berezhiani et al. (1990); Berezhiani \& Khlopov (1990). The extensive review of the results can also be found in the book Khlopov (1997). 
minimal and maximal values of $\sin ^{2}(2 \theta)$ for given $\left.M_{\mathrm{s}}\right)$ is about a factor of 8 .

Taking away the assumptions about the absence of the sterile neutrinos above the temperatures $\sim 1 \mathrm{GeV}$ makes any mixing angle possible. For example, the DM neutrinos can be created due to the inflaton decay Shaposhnikov \& Tkachev (2006). Therefore, in this work we chose not to derive an upper bound on the mass of the sterile neutrino.

Acknowledgements. We would like to thank G. Gilmore, A. Neronov, M. Markevitch, I. Tkachev, and M. Shaposhnikov for help during the various stages of this project. J.N. acknowledges the support from the Academy of Finland. The work of A.B. was (partially) supported by the EU 6th Framework Marie Curie Research and Training network "UniverseNet" (MRTN-CT-2006-035863). The work of O.R. was supported in part by European Research Training Network contract 005104 "ForcesUniverse" and by a Marie Curie International Fellowship within the $6^{\text {th }}$ European Community Framework Programme.

\section{Appendix A: Determining parameters of NFW profile}

Using the data on rotation curves, one usually obtains the following parameters of DM distribution (see e.g. Klypin et al. 2002): virial mass $M_{\text {vir }}$, virial radius $r_{\text {vir }}$, and concentration parameter $C$. They have the following relation with the parameters of NFW profile (5) $r_{\mathrm{s}}$ and $\rho_{\mathrm{s}}$ :

$r_{\mathrm{s}}=\frac{r_{\mathrm{vir}}}{C} ; \quad \rho_{\mathrm{s}}=\frac{M_{\mathrm{vir}}}{4 \pi r_{\mathrm{s}}^{3} f(C)}$,

where in terms of function $f(x)$

$f(x)=\log (1+x)-\frac{x}{1+x}$,

one obtains the mass within the radius $r$ :

$M(r)=M_{\mathrm{vir}} \frac{f\left(r / r_{\mathrm{s}}\right)}{f(C)}$.

If DM distribution in the Milky Way is described by the NFW model (as in Battaglia et al. 2005; Klypin et al. 2002), the flux from a direction $\phi$ is given by

$F_{\mathrm{DM}}^{\mathrm{NFW}}(\phi)=\frac{\Gamma \Omega_{\mathrm{fov}}}{8 \pi} \int_{0}^{\infty} \mathrm{d} z \rho_{\mathrm{NFW}}\left(\sqrt{r_{\odot}^{2}+z^{2}+2 r_{\odot} z \cos \phi}\right)$

(notations are the same as in Eqs. (7), (8)). Let us consider two cases, when the integral in (A.4) can be easily computed. Namely, we have for $\phi=180^{\circ}$

$F_{\mathrm{DM}}^{\mathrm{NFW}}\left(180^{\circ}\right)=\frac{\Gamma \Omega_{\mathrm{fov}}}{8 \pi} \rho_{\mathrm{s}} r_{\mathrm{s}}\left[\log \left(1+\frac{r_{\mathrm{s}}}{r_{\odot}}\right)-\frac{r_{\mathrm{s}}}{r_{\mathrm{s}}+r_{\odot}}\right]$,

and for $\phi=90^{\circ}$

$$
\begin{aligned}
F_{\mathrm{DM}}^{\mathrm{NFW}}\left(90^{\circ}\right)= & \frac{\Gamma \Omega_{\mathrm{fov}}}{8 \pi} \rho_{\mathrm{s}} r_{\mathrm{s}}\left[-1-\log \frac{2 r_{\mathrm{s}}}{r_{\odot}}\right. \\
& \left.+\frac{r_{\odot}^{2}}{r_{\mathrm{s}}^{2}}\left(\frac{3}{2} \log \frac{2 r_{\mathrm{s}}}{r_{\odot}}-\frac{5}{4}\right)\right]+O\left(\frac{r_{\odot}^{4}}{r_{\mathrm{s}}^{4}}\right)
\end{aligned}
$$

(in the latter case the analytic expression is too complicated, so we present Taylor expansion for the case $r_{\odot} \ll r_{\mathrm{s}}$ ).

\section{References}

Abazajian, K. 2006, Phys. Rev. D, 73, 063506

Abazajian, K., \& Koushiappas, S. M. 2006, Phys. Rev. D, 74, 023527

Abazajian, K., Fuller, G. M., \& Patel, M. 2001a, Phys. Rev. D, 64, 023501

Abazajian, K., Fuller, G. M., \& Tucker, W. H. 2001b, ApJ, 562, 593

Aguilar, A., et al. 2001, Phys. Rev. D, 64, 112007

Asaka, T., \& Shaposhnikov, M. 2005, Phys. Lett. B, 620, 17

Asaka, T., Blanchet, S., \& Shaposhnikov, M. 2005, Phys. Lett. B, 631, 151

Asaka, T., Kusenko, A., \& Shaposhnikov, M. 2006a, Phys. Lett. B, 638, 401

Asaka, T., Laine, M., \& Shaposhnikov, M. 2006b, JHEP, 06, 053

Asaka, T., Laine, M., \& Shaposhnikov, M. 2007, JHEP, 01, 091

Barger, V. D., Phillips, R. J. N., \& Sarkar, S. 1995, Phys. Lett. B, 352, 365

Battaglia, G., et al. 2005, MNRAS, 364, 433

Berezhiani, Z. G., \& Khlopov, M. Y. 1990, Sov. J. Nucl. Phys., 52, 60

Berezhiani, Z. G., Vysotsky, M. I., \& Khlopov, M. Y. 1987, Sov. J. Nucl. Phys., 45,1065

Berezhiani, Z. G., Vysotsky, M. I., Yurov, V. P., Doroshkevich, A. G., \& Khlopov, M. Y. 1990, Sov. J. Nucl. Phys., 51, 1020

Biermann, P. L., \& Kusenko, A. 2006, Phys. Rev. Lett., 96, 091301

Bode, P., Ostriker, J. P., \& Turok, N. 2001, ApJ, 556, 93

Boyarsky, A., Neronov, A., Ruchayskiy, O., \& Shaposhnikov, M. 2006a, MNRAS, 370, 213

Boyarsky, A., Neronov, A., Ruchayskiy, O., \& Shaposhnikov, M. 2006b, Phys. Rev. D, 74, 103506

Boyarsky, A., Neronov, A., Ruchayskiy, O., Shaposhnikov, M., \& Tkachev, I. 2006c, Phys. Rev. Lett., 97, 261302

de Gouvea, A. 2005, Phys. Rev. D, 72, 033005

De Luca, A., \& Molendi, S. 2004, A\&A, 419, 837

de Rujula, A., \& Glashow, S. L. 1980, Phys. Rev. Lett., 45, 942

Dodelson, S., \& Widrow, L. M. 1994, Phys. Rev. Lett., 72, 17

Dolgov, A. D., \& Hansen, S. H. 2002, Astropart. Phys., 16, 339

Doroshkevich, A. G., Khlopov, M. I., \& Klypin, A. A. 1989, MNRAS, 239, 923

Ghigna, S., Moore, B., Governato, F., et al. 2000, ApJ, 544, 616

Gilmore, G., Wilkinson, M., Kleyna, J., et al. 2006

[arXiv: astro-ph/0608528]

Gilmore, G., et al. 2007 [arXiv: astro-ph/0703308]

Goerdt, T., Moore, B., Read, J. I., Stadel, J., \& Zemp, M. 2006, MNRAS, 368, 1073

Hansen, S. H., Lesgourgues, J., Pastor, S., \& Silk, J. 2002, MNRAS, 333, 544

Hickox, R. C., \& Markevitch, M. 2006, ApJ, 645, 95

Hidaka, J., \& Fuller, G. M. 2006 [arXiv:astro-ph/0609425]

Khlopov, M. Y. 1997, Cosmoparticle Physics (World Scientific Pub Co Inc)

Kleyna, J. T., Wilkinson, M. I., Gilmore, G., \& Evans, N. W. 2003, ApJ, 588, L21

Klypin, A., Zhao, H., \& Somerville, R. S. 2002, ApJ, 573, 597

Kusenko, A. 2006a [arXiv: astro-ph/0609375]

Kusenko, A. 2006b, Phys. Rev. Lett., 97, 241301

Mateo, M. L. 1998, ARA\&A, 36, 435

Navarro, J. F., Frenk, C. S., \& White, S. D. M. 1997, ApJ, 490, 493

Nevalainen, J., Bonamente, M., \& Kaastra, J. 2006

[arXiv: astro-ph/0610461]

Nevalainen, J., Markevitch, M., \& Lumb, D. 2005, ApJ, 629, 172

Pal, P. B., \& Wolfenstein, L. 1982, Phys. Rev. D, 25, 766

Riemer-Sørensen, S., Hansen, S. H., \& Pedersen, K. 2006a, ApJ, 644, L33

Riemer-Sørensen, S., Pedersen, K., Hansen, S. H., \& Dahle, H. 2006b [arXiv: astro-ph/0610034]

Seljak, U., Makarov, A., McDonald, P., \& Trac, H. 2006, Phys. Rev. Lett., 97, 191303

Shaposhnikov, M., \& Tkachev, I. 2006, Phys. Lett. B, 639, 414

Shi, X.-d., \& Fuller, G. M. 1999, Phys. Rev. Lett., 82, 2832

Stasielak, J., Biermann, P. L., \& Kusenko, A. 2006

[arXiv: astro-ph/0606435]

Strigari, L. E., et al. 2006 [arXiv: astro-ph/0603775]

Strumia, A., \& Vissani, F. 2006, hep-ph/0606054

Viel, M., Lesgourgues, J., Haehnelt, M. G., Matarrese, S., \& Riotto, A. 2005, Phys. Rev. D, 71, 063534

Viel, M., Lesgourgues, J., Haehnelt, M. G., Matarrese, S., \& Riotto, A. 2006, Phys. Rev. Lett., 97, 071301

Wargelin, B. J., Markevitch, M., Juda, M., et al. 2004, ApJ, 607, 596

Watson, C. R., Beacom, J. F., Yuksel, H., \& Walker, T. P. 2006, Phys. Rev. D, 74, 033009

Wilkinson, M. I. et al. 2006 [arXiv: astro-ph/0602186]

Wu, X. 2007 [arXiv:astro-ph/0702233] 